\title{
Mitteilungen der Arbeitsgemeinschaft für prächirurgische Diagnostik und operative Epilepsietherapie
}

Z. Epileptol. 2020 ·33:182

https://doi.org/10.1007/s10309-020-00331-x

() Springer Medizin Verlag GmbH, ein Teil von Springer Nature 2020

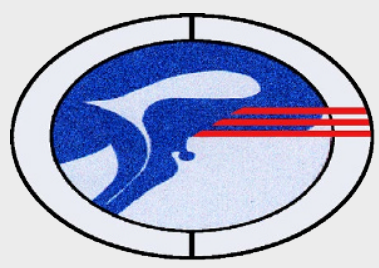

AG Epilepsiechirurgie
Korrespondenzadresse/Redaktion

Prof. Dr. med. Adam Strzelczyk, MHBA (V.i.S.d.P.)

Sekretär der AG Epilepsiechirurgie

Epilepsiezentrum Frankfurt Rhein-Main

Universitätsklinikum Frankfurt

Schleusenweg 2-16, 60528 Frankfurt

strzelczyk@med.uni-frankfurt.de

Arbeitsgemeinschaft für prächirurgische Diagnostik und operative Epilepsietherapie e.V. www.ag-epilepsiechirurgie.de

\section{ACHTUNG: Termin- und Ortsänderung}

\section{Einladung zur Jahrestagung der Arbeitsgemeinschaft für prächirurgische Diagnostik und operative Epilep- sietherapie e.V. im Rahmen der 55. Jahrestagung der Deutschen Gesellschaft für Epileptologie in Freiburg im Breisgau 2020}

\begin{abstract}
Die geplante Jahrestagung der AG Epilepsiechirurgie am 19.09.2020 in Wien wird wegen der Corona Pandemie ausfallen. Die Jahrestagung 2020 und anschließende Mitgliederversammlung findet dieses Jahr am 09.09.2020 in Freiburg im Breisgau statt. Wir freuen uns sehr auf Ihre Teilnahme an der Veranstaltung und den Diskussionen.

Die nächste Jahrestagung der AG Epilepsiechirurgie wird entsprechend in Wien für Sommer 2021 geplant.
\end{abstract}

Für den Vorstand der AG

Ihre

\section{Prof. Martha Feucht \\ Erste Vorsitzende}

\section{Prof. Dr. Adam Strzelczyk}

Sekretär

\section{Programm der Jahrestagung der Arbeitsgemeinschaft für prächirurgische Diagnostik und operative Epilepsie- therapie e.V.}

\section{Zeit: Mittwoch, 09.09.2020 8.15 bis 9.45}

Adresse: Konzerthaus Freiburg, Konrad-Adenauer-Platz 1, D-79098 Freiburg im Breisgau

- Therapieresistente Epilepsie und hemisphärale Pathologie im MRT: Stellenwert der prächirurgischen Diagnostik - Martha Feucht (Wien/AT)

- Maximal- bis minimal-invasive Therapieverfahren bei Säuglingen und Kleinkindern - Marec von Lehe (Neuruppin/D)

- Chirurgische Aspekte invasiver Ableitungen in den ersten Lebensjahren - Christian Dorfer (Wien/AT)
- ECoG und Hochfrequenz Oszillationen während Epilepsiechirurgie bei Kindern - Johannes Sarnthein (Zürich/CH)

- Einflussfaktoren auf das postoperative neuropsychologische Outcome bei Kindern und Jugendlichen - Thomas Cloppenburg (Bielefeld/D)

\section{Einladung zur Mitgliederversammlung}

\section{Tagesordnung der Mitgliederversammlung der Arbeitsgemeinschaft für prächirurgische Diagnostik und operative Epilepsietherapie e.V.}

Zeit: Mittwoch 09.09.2020, von 9.45 - ca. 10.15 Uhr

Adresse: Konzerthaus Freiburg, Konrad-Adenauer-Platz 1, D-79098 Freiburg im Breisgau

TOP 1) Begrüßung und Feststellung der Beschlussfähigkeit

TOP 2) Bestätigung der Tagesordnung

TOP 3) Bestätigung des Protokolls der letzten Mitgliederversammlung

TOP 4) Bericht der 1. Vorsitzenden, Prof. Martha Feucht, Wien

TOP 5) Bericht des 2. Vorsitzenden, Prof. Dr. Hans-Jürgen Huppertz, Zürich

TOP 6) Bericht des Sekretärs, Prof. Dr. Adam Strzelczyk, Frankfurt am Main

TOP 7) Bericht des Schatzmeisters, PD Dr. Volkmar Hans, Neubrandenburg

TOP 8) Berichte der Kassenprüfer

TOP 9) Tagungsort und Tagungsthema Wien 2021

TOP 10) Entlastung des Vorstandes

TOP 11) Update 3. Kohorte des Mini-Fellowships Epilepsiechirurgie TOP 12) Verschiedenes

gez.

Prof. Dr. med. Adam Strzelczyk

Sekretär der AG 